\title{
Investigating Natural Red Lakes with Two-Photon Excited Fluorescence Lifetime Imaging Microscopy
}

\author{
Tana Elizabeth Villafana ${ }^{1,2}$, Ryan Beams ${ }^{2}$, John K. Delaney ${ }^{3}$, Fenella France ${ }^{1}$, and Stephan J. Stranick ${ }^{2}$ \\ 1. Preservation Research and Testing Division, Library of Congress, Washington, DC, USA \\ 2. Material Measurement Science Division, National Institute of Standards and Technology, \\ Gaithersburg, MD, USA \\ 3. Scientific Research Department, National Gallery of Art, Washington, DC, USA
}

Insect or plant sourced organic red dyes are present in many historic artifacts, such as illuminated (painted) manuscripts, paintings, textiles, and works on paper, typically in the form of a lake (extracted dye precipitated onto a metal cation or salt) [1]. Identification of these lakes, both the dye and its substrate can provide information on dating, provenance, and authenticity as well as guide conservation and preservation treatments. Significant effort has been devoted to characterizing organic lakes with both invasive and non-invasive techniques explored [2], yet accurate non-destructive identification remains an analytical challenge. In general, non-invasive spectroscopic techniques are complicated by the dependence of the lake's optical properties on dye concentration, surrounding environment (media, other pigments or chromophores), $\mathrm{pH}$, metal substrate, and even the original extraction and metal precipitation procedure for the dye [3].

In this paper we use two-photon excited fluorescence lifetime imaging microscopy (2p-FLIM) to effectively distinguish between model samples of madder, carmine, and lac lakes, in acrylic painted films, independent of the pigment to binder ratio. Lifetimes for our (lab-prepared) red-pigmented acrylic paint films were found to follow a tri-exponential decay behavior with stark differences in the second lifetime component, $\tau_{2}$, for madder $(\approx 2.5 \mathrm{~ns})$, lac $(<100 \mathrm{ps})$, and carmine $(\approx 0.5 \mathrm{~ns})$ lakes. As seen in figure 1a, image contrast based on $\tau_{2}$ immediately reveals that these three layers are composed of different materials, though the bright field image shows three relatively similar red paint films. These lifetime differences are highlighted in the decay plots (figure 1b).

Our initial lifetime results are in stark contrast with other lifetimes reported for carmine, madder, and lac. Bulk linear fluorescence lifetime measurements $\left(\tau_{1}, \tau_{2}\right)$ were performed on madder, carmine, and lac lakes in oil painted films and reported as (1.2 ns, $4.3 \mathrm{~ns}),(1.5 \mathrm{~ns}, 5.0 \mathrm{~ns})$, and (0.9 ns, $2.6 \mathrm{~ns})$, respectively,[4] which decays much slower than our measured lifetimes (although the trend of lac having a shorter lifetime than carmine is the same). This is most likely because a bulk lifetime measurement technique is highly affected by scattering and self-absorption; higher concentrations and more scattering causes a lengthening in measured fluorescence lifetimes [5]. One of the largest advantages of nonlinear microscopy is that the signals are weakly affected by scattering [6]. Figure 2 shows that we measured consistent lifetime decay behavior for madder, carmine, and lac lakes, regardless of pigment concentration, ranging from $0.05 \mathrm{wt} \%$ to $25 \mathrm{wt} \%$ in acrylic.

As these results show, 2p-FLIM is a promising technique for non-invasive differentiation and identification of organic red paints noninvasively, opening the way to studies of real-world samples. Ongoing work includes investigating a series of lap-prepared sample sets on different supports in different binding media, including tracking the effect of aging and conservation treatments on the baseline lifetimes of these samples. 
References:

[1] J Kirby, R White, National Gallery Technical Bulletin 1996, 17, 56-80.

[2] I Degano et al, Applied Spectroscopy Reviews 44 (2009), pg. 363-410.

[3] C Clementi et al, Applied Physics A 92 (2008), pg. 25-33.

[4] A. Romani et al, Applied spectroscopy 62 (2008), pg. 1395-1399.

[5] S. P. Barbosa et al, European Conference on Biomedical Optics (2003), pg. 5138-5310.

[6] W. R. Zipfel, R. M. Williams, W. W. Webb, Nat Biotechnol 21 (2003), pg. 1369-1377.
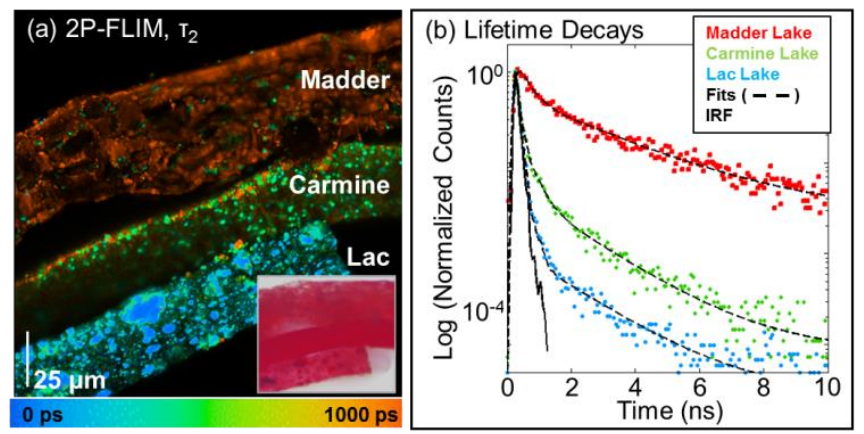

Figure 1. : (a) $\tau_{2} 2 \mathrm{P}-F L I M$ image $(1.5 \mathrm{~mW}$ average power, $810 \mathrm{~nm}$, scan range $175 \mathrm{x} 175 \mu \mathrm{m}, 512 \mathrm{x}$ 512 pixels, $50 \mathrm{~ms}$ pixel dwell time, intensity scaling of 5000 photons per pixel) and (inset) bright field image of a cross-section (cropped to $175 \times 175 \mu \mathrm{m})$ composed of madder (10 wt \%), cochineal (2 wt \%), and lac (2 wt \%). (b) The two-photon fluorescence signals correspond to pigment particles in carmine and lac layers, whereas in madder, the signal appears to be spatially related to acrylic as opposed to madder particles.

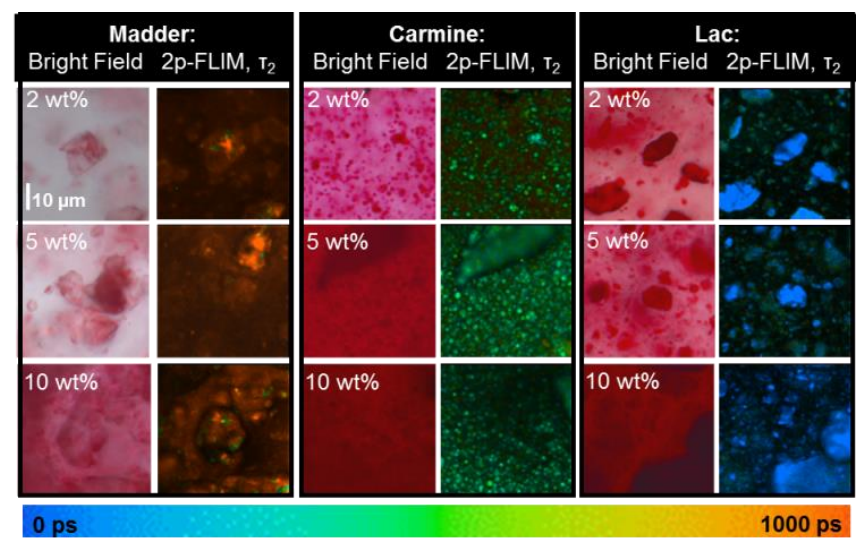

Figure 2. Bright field and corresponding $\tau_{2} 2 p$-FLIM images of madder (left column), carmine (middle column), and lac (right column) lakes, with concentrations of each indicated in the bright field image and color-coding of lifetime at right of each column. Lifetimes at 0 ps will be blue with longer lifetimes going from cyan, green, yellow, and orange to red. Any lifetimes longer than $1000 \mathrm{ps}$ will appear red in the images. Comparison of 2p-FLIM images (from Figure 1) indicate that in carmine and lac the $2 \mathrm{p}$ FLIM signal is originating from the pigment particles while the lifetime images show that changes in concentration have no effect on lifetimes of these particles. 